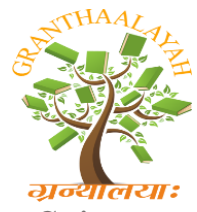

Science

\section{INTERNATIONAL JOURNAL OF RESEARCH - GRANTHAALAYAH A knowledge Repository}

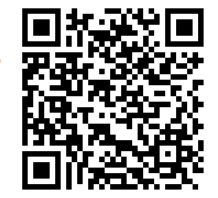

\title{
A STUDY ON POLYNOMIAL TERNARY SEMI RING
}

T. Srinivasa Rao ${ }^{1}$, Dr. D. Madhusudana Rao*2, B. Srinivasa Kumar ${ }^{3}$

1, 3 Department of Mathematics, K L University, Vaddeswaram, Guntur Dt., INDIA

${ }^{* 2}$ Lecturer and Head, Department of Mathematics, VSR\&NVR College, Tenali, A.P, INDIA

\section{ABSTRACT}

In this paper we discussed about the polynomial ternary semiring and characterized them.

\section{Mathematical Subject Classification : 16Y30, 16 Y99}

\section{Keywords:}

Polynomial Ternary Semiring, Commutative polynomial ternary semiring, Quasi Commutative polynomial ternary Semiring, Normal, pseudo Commutative.

Cite This Article: T. Srinivasa Rao, Dr. D. Madhusudana Rao, and B. Srinivasa Kumar, "A STUDY ON POLYNOMIAL TERNARY SEMI RING" International Journal of Research - Granthaalayah, Vol. 3, No. 8(2015): 99-104. DOI: 10.29121/granthaalayah.v3.i8.2015.2964.

\section{INTRODUCTION}

Algebraic structures play a prominent role in mathematics with wide ranging applications in many disciplines such as theoretical physics, computer sciences, control engineering, information sciences, coding theory, topological spaces, and the like. The theory of ternary algebraic systems was introduced by D. H. Lehmer [2]. He investigated certain ternary algebraic systems called triplexes which turn out to be commutative ternary groups. D. Madhusudhana Rao[3] characterized the primary ideals in ternary semigroups. About T. K. Dutta and S. Kar [1] introduced and studied some properties of ternary semirings which is a generalization of ternary rings. Our main purpose in this paper is to introduce the notion of some special class of ternary semirings.

\section{PRELIMINARIES}

Definition 2.1 : A nonempty set $\mathrm{T}$ together with a binary operation called addition and a ternary multiplication denoted by [ ] is said to be a ternary semiring if $\mathrm{T}$ is an additive commutative semigroup satisfying the following conditions :

i) $[[a b c] d e]=[a[b c d] e]=[a b[c d e]]$,

ii) $[(a+b) c d]=[a c d]+[b c d]$,

iii) $[a(b+c) d]=[a b d]+[a c d]$,

iv) $[a b(c+d)]=[a b c]+[a b d]$ for all $a ; b ; c ; d ; e \in \mathrm{T}$. 
Throughout $\mathrm{T}$ will denote a ternary semiring unless otherwise stated.

Note 2.2: For the convenience we write $x_{1} x_{2} x_{3}$ instead of $\left[x_{1} x_{2} x_{3}\right]$

Note 2.3 : Let $\mathrm{T}$ be a ternary semiring. If $\mathrm{A}, \mathrm{B}$ and $\mathrm{C}$ are three subsets of $\mathrm{T}$, we shall denote the set $\mathrm{ABC}=\{\Sigma a b c: a \in A, b \in B, c \in C\}$.

Note 2.4 : Let $\mathrm{T}$ be a ternary semiring. If $\mathrm{A}, \mathrm{B}$ are two subsets of $\mathrm{T}$, we shall denote the set $\mathrm{A}+\mathrm{B}=\{a+b: a \in A, b \in B\}$.

Note 2.5 : Any semiring can be reduced to a ternary semiring.

Example 2.6 : Let $\mathrm{T}$ be an semigroup of all $m \times n$ matrices over the set of all non negative rational numbers. Then $\mathrm{T}$ is a ternary semiring with matrix multiplication as the ternary operation.

\section{MAIN RESULTS}

Definition 3.1: Let $\mathrm{T}$, be a Ternary Semi ring. The set $\mathrm{P}[x]$ of all polynomials over a Ternary semi ring, $\mathrm{T}$ is said to be Polynomial Ternary Semiring(denoted by $\mathrm{P}_{\mathrm{T}}[x]$ ) with respect to addition + and Ternary multiplication [ ] of the polynomials provided

$[(f g h) i j]=[f(g h i) j]=[f g(h i j)]$

$[(f+g) h i]=[f h i]+[g h i]$

$[f(g+h) i]=[f g i]+[f h i]$

$[f g(h+i)]=[f g h]+[f g i]$

$(f+g) \in \mathrm{P}[x], \forall f, g, h, i, j \in \mathrm{P}[x]$.

Note 3.2: Let $P_{T}[x]$ be the polynomial ternary semiring, then the operations addition and multiplication on $\mathrm{P}[\mathrm{x}]$ is defined as follows:.

For any $f, g, h \in \mathrm{P}[x]$ where,

$$
\begin{aligned}
f=f(x)=a_{0}+a_{1} x+ & a_{2} x^{2}+\cdots+a_{n} x^{n} \\
& g=g(x)=b_{0}+b_{1} x+b x^{2}+\cdots+b x^{n} \\
h & =h(\mathrm{x})=c_{0}+c x+c x^{2}+\cdots+c x^{n}
\end{aligned}
$$

Then, $f+g=e_{0}+e x+e_{2} x^{2}+\cdots+e_{n} x^{n}, e_{0}=a_{0}+b_{0}, e_{1}=a_{1}+b_{1}, e_{2}=a_{2}+b_{2},+\cdots$ Also f.g.h $=\mathrm{f}(\mathrm{x}) \cdot \mathrm{g}(\mathrm{x}) \cdot \mathrm{h}(\mathrm{x})=d_{0}+d_{1} x+d_{2} x^{2}+\cdots+d_{n} x^{n}$, where $d_{n}=\sum_{i+j+k}\left(a_{i} b_{j} c_{k}\right)$ And also $d_{1}=d_{3}=\ldots+d_{2 n-1} \neq 0, \quad d_{2}=d_{4}=\ldots d_{2 n}=0$.

Example 3.3: The set of all polynomials over real numbers forms a Ternary polynomial semi ring $\mathrm{P}_{\mathrm{T}}[\mathrm{x}]$ by using polynomial addition and Ternary multiplication.

Example 3.4: The set $\mathrm{P}[\mathrm{x}]$ consisting of a single polynomial $\mathrm{o}[x]$ with binary operation defined $0+0=0$ and Ternary multiplication defined by $0.0 .0=0$ is a Ternary polynomial semi ring, $\mathrm{P}_{\mathrm{T}}[x]$ this ring is called the Null polynomial ternary semi ring.

Definition 3.5: A ternary polynomial semi ring, $\mathrm{P}_{\mathrm{T}}[\mathrm{x}]$ is said to be a Commutative polynomial Ternary semi ring if $[f g h]=[g h f]=[h f g]=[f h g]=[g h f]=[h g f], \forall f, g, h \in \mathrm{P}_{\mathrm{T}}[x]$. Where,

$$
\begin{aligned}
f=f(x)=a_{0}+a_{1} x+ & a_{2} x^{2}+\cdots+a_{n} x^{n} \\
& g=g(x)=b_{0}+b_{1} x+b x^{2}+\cdots+b x^{n} \\
h=h(x) & =c_{0}+c x+c x^{2}+\cdots+c x^{n}
\end{aligned}
$$

Then $\quad \sum_{l+m+k=n}\left(a_{l} b_{m} c_{k}\right)=\sum_{l+m+k=n}\left(b_{m} c_{k} l\right)=\sum_{l+m+k=n}\left(c_{k} a_{l} b_{m}\right) \quad$ and also $\sum_{l+m+k=n}\left(a_{l} b_{m} c_{k}\right), \sum_{l+m+k=n}\left(b_{l} c_{m} a_{k}\right), \sum_{l+m+k=n}\left(c_{l} a_{m} b_{k}\right)$.

Example 3.6: A polynomial ternary semi ring $\mathrm{P}_{\mathrm{T}}[\mathrm{x}]$ over the set of Complex numbers, $\mathrm{C}$ is a Commutative Ternary polynomial semi ring. 
Definition 3.7: A polynomial ternary semi ring, $\mathrm{P}_{\mathrm{T}}[\mathrm{x}]$ is said to be a Quasi Commutative polynomial ternary semi ring if $[f g h]=\left[g^{n} f h\right]=[g h f]=\left[h^{n} g f\right]=[h f g]=\left[f^{n} h g\right], \forall f, g, h \in \mathrm{P}_{\mathrm{T}}[x]$ and $n$ must be an odd natural number.

Example 3.8 : A polynomial ternary semi ring $\mathrm{P}_{\mathrm{T}}[x]$ over the set of Complex numbers, $\mathrm{C}$ is a Quasi Commutative Ternary polynomial semi ring.

Theorem 3.9: A polynomial ternary semi ring $\mathrm{P}_{\mathrm{T}}[x]$ is commutative iff it is quasi commutative Ternary polynomial semi ring.

Proof: Let $\mathrm{P}_{\mathrm{T}}[\mathrm{x}]$ be a Commutative Ternary polynomial semi ring.

We have to show that $\mathrm{P}_{\mathrm{T}}[\mathrm{x}]$ be a quasi-commutative Ternary polynomial semi ring.

for any $\mathrm{f}, \mathrm{g}, \mathrm{h} \in \mathrm{P}_{\mathrm{T}}[\mathrm{x}]$, where

$$
\begin{aligned}
f=f(x)=a_{0}+a_{1} x+ & a_{2} x^{2}+\cdots+a_{n} x^{n} \\
& g=g(x)=b_{0}+b_{1} x+b x^{2}+\cdots+b x^{n} \\
& h=h(x)=c_{0}+c x+c x^{2}+\cdots+c x^{n} .
\end{aligned}
$$

since $\mathrm{P}_{\mathrm{T}}[x]$ be a Commutative polynomial ternary semi ring by def.

$[f g h]=[g h f]=[h f g]=[f h g]=[g h f]=[h g f], \forall f, g, h \in \mathrm{P}_{\mathrm{T}}[x]$

we can also observe, $[f g h]=\left[g^{l} h f\right]=[h f g]=\left[f^{l} h g\right]=[g h f]=\left[h^{1} g f\right]$

$[f g h]=\left[g^{n} h f\right]=[h f g]=\left[f^{n} h g\right]=[g h f]=\left[h^{n} g f\right]$ this true for every odd natural number, $n$.

Therefore, $\mathrm{P}_{\mathrm{T}}[x]$ is a quasi commutative Ternary polynomial semi ring.

Conversely suppose that $\mathrm{P}_{\mathrm{T}}[x]$ be a quasi commutative Ternary polynomial semi ring.

Now we have to verify $\mathrm{P}_{\mathrm{T}}[x]$ be a Commutative Ternary polynomial semi ring.

by the definition of quasi commutative $[f g h]=[g h f]=[h f g]=\left[f^{n} h g\right]=\left[g^{n} h f\right]=[\mathrm{i}]$, for some odd natural $n$ and $f, g, h \in \mathrm{P}_{\mathrm{T}}[x]$.

$[f g h]=\left[g^{l} h f\right]=[h f g]=\left[f^{l} h g\right]=[g h f]=\left[h^{l} g f\right], \forall f, g, h \in \mathrm{P}_{\mathrm{T}}[x]$.

Therefore $\mathrm{P}_{\mathrm{T}}[x]$ be a commutative $x$ polynomial ternary semi ring.

Definition 3.10: A polynomial ternary semi ring, $\mathrm{P}_{\mathrm{T}}[\mathrm{x}]$ is said to be a normal polynomial ternary semi ring if $f g . \mathrm{P}_{\mathrm{T}}[x]=\mathrm{P}_{\mathrm{T}}[x] . f g, \forall f, g \in \mathrm{P}_{\mathrm{T}}[x]$.

Example 3.11: A polynomial ternary semi ring, $\mathrm{P}_{\mathrm{T}}[x]$ over set of Complex numbers, $\mathrm{C}$ is a normal polynomial ternary semi ring.

Theorem 3.12. A polynomial ternary semi ring $\mathrm{P}_{\mathrm{T}}[x]$ is quasi commutative iff it is normal polynomial ternary semi ring.

Proof: Let $\mathrm{P}_{\mathrm{T}}[x]$ be a Commutative polynomial ternary semi ring.

by definition of quasi commutative for any $f, g, h \in \mathrm{P}_{\mathrm{T}}[x]$,

$[f g h]=[g h f]=[h f g]=\left[f^{n} h g\right]=\left[g^{n} h f\right]=\left[h^{n} g f\right]$, for some odd natural $n$.

let, $k \in f g . \mathrm{P}_{\mathrm{T}}[x] \Rightarrow k=[f g h]$, for some $h \in \mathrm{P}_{\mathrm{T}}[x]$.

since $\mathrm{P}_{\mathrm{T}}[x]$ is quasi then, $k=[f g h]=\left[h^{n} g f\right]=[h f g]$, where $h, f, g \in \mathrm{P}_{\mathrm{T}}[x] . f g$

therefore, $k \in f g . \mathrm{P}_{\mathrm{T}}[x] \Rightarrow \mathrm{k} \in \mathrm{P}_{\mathrm{T}}[\mathrm{x}] . \mathrm{fg}$

therefore $f g . \mathrm{P}_{\mathrm{T}}[x]$ is contained in $\mathrm{P}_{\mathrm{T}}[x] . f g$

similarly we can show that $\mathrm{P}_{\mathrm{T}}[x] . f g$ is contained in $f g . \mathrm{P}_{\mathrm{T}}[x]$.

Therefore $f g . \mathrm{P}_{\mathrm{T}}[x]=\mathrm{P}_{\mathrm{T}}[x] . f g, \forall f, g \in \mathrm{P}_{\mathrm{T}}[x]$.

Conversely suppose that $\mathrm{P}_{\mathrm{T}}[x]$ is a normal Ternary polynomial semi ring.

We have to show $\mathrm{P}_{\mathrm{T}}[x]$ is quasi Ternary polynomial semi ring.

by the definition of normal for any $f, g, h \in \mathrm{P}_{\mathrm{T}}[x]$, then $f g \cdot \mathrm{P}_{\mathrm{T}}[x]=\mathrm{P}_{\mathrm{T}}[x] . f g$

$\Rightarrow f . g . h=h . f . g$ for any $h \in \mathrm{P}_{\mathrm{T}}[x] \Rightarrow f \cdot g \cdot h^{n}=h^{n} . f \cdot g$ for any $h \in \mathrm{P}_{\mathrm{T}}[x]$, when $n=1$ 
$\Rightarrow$ This is true for any odd natural number $n$.

Therefore $\mathrm{P}_{\mathrm{T}}[x]$ is quasi polynomial ternary semi ring. Hence the theorem is proved.

Corollary 3.13: Every commutative polynomial ternary semi ring is quasi commutative Ternary polynomial ring.

Corollary 3.14: Every quasi commutative polynomial ternary semi ring is normal commutative polynomial ternary semi ring.

Definition 3.15: A polynomial ternary semi ring, $\mathrm{P}_{\mathrm{T}}[x]$ is said to be left pseudo commutative polynomial ternary semi ring if $[(f g h) i j]=[(g h f) i j]=[(h f g) i j]=[(f h g) i j]=[(g f h) i j]=[(h g f) i j], \forall$ $f, g, h, i, j \in \mathrm{P}_{\mathrm{T}}[x]$.

Example 3.16: A polynomial ternary semi ring, $\mathrm{P}_{\mathrm{T}}[x]$ over set of Complex numbers, $\mathrm{C}$ is left pseudo commutative Ternary semi ring.

Theorem 3.17: If $\mathrm{P}_{\mathrm{T}}[x]$ is a commutative polynomial ternary semi ring then $\mathrm{P}_{\mathrm{T}}[x]$ is left pseudo commutative polynomial ternary semi ring.

Proof: Let $\mathrm{P}_{\mathrm{T}}[x]$ is a commutative polynomial ternary semi ring.

By definition for any $f, g, h \in \mathrm{P}_{\mathrm{T}}[x] \Rightarrow[f g h]=[g h f]=[h f g]=[f h g]=[g h f]=[h g f]$

$\Rightarrow$ for any $i, j \in \mathrm{P}_{\mathrm{T}}[x],[(f g h) i j]=[(g h f) i j]=[(h f g) i j]=[(f h g) i j]=[(g f h) i j]=[(h g f) i j]$.

Therefore $\mathrm{P}_{\mathrm{T}}[x]$ is left pseudo commutative Ternary semi ring.

Note 3.18: The converse of the above theorem is need not be true.

Definition 3.19 : A polynomial ternary semi ring, $\mathrm{P}_{\mathrm{T}}[x]$ is said to be lateral pseudo commutative polynomial ternary semi ring if $[(f(g h i) j]=[f(h i g) j]=[f(i g h) j]=[(f(g i h) j]$ $=[f(h g i) j]=[f(i h g) j], \forall f,, g, h, I, j \in \mathrm{P}_{\mathrm{T}}[x]$.

Definition 3.20: A polynomial ternary semi ring, $\mathrm{P}_{\mathrm{T}}[x]$ is said to be right pseudo commutative polynomial ternary semi ring if $[f g(h i j)]=[f g(i j h)]=[f g(j h i)]=[f g(h j i)]=[f g(i h j)]=[f g(j i h)], \forall$ $f, g, h, I, j \in \mathrm{P}_{\mathrm{T}}[x]$.

Theorem 3.21. If $\mathrm{P}_{\mathrm{T}}[x]$ is a commutative polynomial ternary semi ring then it is lateral pseudo commutative polynomial ternary semi ring.

Proof: by the definition of commutative polynomial ternary semi ring, for any $f, g, h \in \mathrm{P}_{\mathrm{T}}[x]$

$[f g h]=[g h f]=[h f g]=[f h g]=[g h f]=[h g f]$.

For any $i, j \in \mathrm{P}_{\mathrm{T}}[x], \Rightarrow[i(f g h) j]=[i(g h f) j]=[i(h f g) j]=[i(f h g) j]=[i(g f h) j]=[i(h g f) j]$

$\Rightarrow \mathrm{P}_{\mathrm{T}}[x]$ is lateral pseudo commutative polynomial ternary semi ring.

Corollary 3.22: If $\mathrm{P}_{\mathrm{T}}[\mathrm{x}]$ is a commutative Ternary polynomial semi ring then it is left and right pseudo commutative Ternary semi ring.

Corollary 3.23: The converse of the above theorem is need not be true.

Definition 3.24: A polynomial ternary semi ring, $\mathrm{P}_{\mathrm{T}}[x]$ is said to be pseudo commutative polynomial ternary semi ring if it is left and right pseudo commutative polynomial ternary semi ring. 
Theorem 3.25: Every Ternary polynomial semi ring, $P_{\mathrm{T}}[\mathrm{x}]$ is pseudo commutative Ternary semi ring.

Proof: By theorem 3.17, theorem 3.21, and corollary 3.22, a polynomial ternary semi ring, $\mathrm{P}_{\mathrm{T}}[x]$ is left, right and lateral pseudo polynomial ternary semi ring. Hence the theorem.

Definition 3.26: if $\mathrm{P}_{\mathrm{T}}[x]$, is a polynomial ternary semi ring, and $\mathrm{S}_{\mathrm{T}}[x]$ is a non-empty sub set of $\mathrm{P}_{\mathrm{T}}[x]$ then $\mathrm{S}_{\mathrm{T}}[x]$ is said to be a polynomial sub ternary semi ring if $\mathrm{S}_{\mathrm{T}}[x]$ is a polynomial ring under the addition and Ternary multiplication defined on $\mathrm{P}_{\mathrm{T}}[x]$.

Example 3.27: $\mathrm{S}_{\mathrm{Z}}[x]$, the set of polynomials over the set of integers is a polynomial sub ternary semi ring of $\mathrm{P}_{\mathrm{R}}[x]$, the polynomial sub ternary semi ring over the set of real numbers.

Theorem 3.28: The non-empty intersection of two polynomial sub ternary semi ring is again a polynomial ternary sub semi ring.

Proof: Let $\mathrm{S}_{\mathrm{P}}[x]$ and $\mathrm{S}_{\mathrm{Q}}[x]$ be two polynomial sub ternary semi ring of $\mathrm{P}_{\mathrm{T}}[\mathrm{x}]$, where $\mathrm{P}$ and $\mathrm{Q}$ are ternary sub semiring of $\mathrm{T}$.

For any $f, g, h \in \mathrm{S}_{\mathrm{P}}[x] \cap \mathrm{S}_{\mathrm{Q}}[x] \Rightarrow f, g, h \in \mathrm{S}_{\mathrm{P}}[\mathrm{x}]$ and $f, g, h \in \mathrm{S}_{\mathrm{Q}}[x]$.

Since $\mathrm{S}_{\mathrm{P}}[x]$ and $\mathrm{S}_{\mathrm{Q}}[x]$ are polynomial sub ternary semi rings then

$f+g \in \mathrm{S}_{\mathrm{P}}[x]$ and $f+g \in \mathrm{S}_{\mathrm{Q}}[x] \Rightarrow f+g \in \mathrm{S}_{\mathrm{P}}[x] \cap \mathrm{S}_{\mathrm{Q}}[x]$

and also by ternary multiplication, f.g.h $\in \mathrm{S}_{\mathrm{P}}[x]$ and $f . g . h \in \mathrm{S}_{\mathrm{Q}}[x] \Rightarrow f . g . h \in \mathrm{S}_{\mathrm{P}}[x] \cap \mathrm{S}_{\mathrm{Q}}[x]$

Therefore $\mathrm{S}_{\mathrm{P}}[x] \cap \mathrm{S}_{\mathrm{Q}}[x]$ is a polynomial sub ternary semi ring.

Definition 3.29: Let $\mathrm{S}_{\mathrm{T}}[x]$ is a non-empty sub set of a polynomial ternary semi ring of $\mathrm{P}_{\mathrm{T}}[\mathrm{x}]$. The smallest polynomial ternary sub semi ring of $\mathrm{P}_{\mathrm{T}}[x]$ containing $\mathrm{S}_{\mathrm{T}}[x]$ is called a polynomial sub ternary semiring of $P_{\mathrm{T}}[x]$ generated by $S_{\mathrm{T}}[x]$. It is denoted by $\left\langle\mathrm{S}_{\mathrm{T}}[x]\right\rangle$.

Theorem 3.30: $P_{\mathrm{T}}[x]$ is a polynomial ternary semi ring and $\mathrm{S}_{\mathrm{T}}[x]$ is a polynomial sub ternary semi ring of $\mathbf{P}_{\mathbf{T}}[x]$ then $\left\langle\mathbf{S}_{\mathbf{T}}[\boldsymbol{x}]\right\rangle=\left\{f_{1} \cdot f_{2} . f_{3} .---. f_{n} / f_{1}, f_{2}, f_{3}, \ldots f_{n} \in S_{T}[x]\right\}$.

Proof: Let $\left\langle\mathrm{K}_{\mathrm{T}}[x]>=\left\{f_{1} \cdot f_{2} \cdot f_{3} .---f_{n} / f_{1}, f_{2}, f_{3}, \ldots f_{n} \in S_{T}[x]\right\}\right.$.

Let $r, s, t \in \mathrm{K}_{\mathrm{T}}[x]$, where

$$
\begin{aligned}
r=r(x)=r_{1} \cdot r_{2} \cdot r_{3} \cdot-- & -r_{n} \\
s & =s(x)=s_{1} \cdot s_{2} \cdot s_{3} \cdot---\cdot s_{n} \\
t & =t(x)=t_{1} \cdot t_{2} \cdot t_{3} \cdot---t_{n} .
\end{aligned}
$$

where $r_{1} \cdot r_{2} \cdot r_{3} \cdot---. r_{n}, s_{1} \cdot s_{2} \cdot s_{3} \cdot---. s_{n}, t_{1} \cdot t_{2} \cdot t_{3} \cdot---t_{n} \in S_{T}[x]$

now r.s.t $=\left(r_{1} \cdot r_{2} \cdot r_{3} \cdot---r_{n}\right)\left(s_{1} \cdot s_{2} \cdot s_{3} \cdot---s_{n}\right)\left(t_{1} \cdot t_{2} \cdot t_{3} \cdot--\cdot t_{n}\right) \in \mathrm{K}_{\mathrm{T}}[x]$.

$\Rightarrow$ r.s.t $\mathrm{K}_{\mathrm{T}}[x] \Rightarrow \mathrm{K}_{\mathrm{T}}[x]$ is a Ternary sub polynomial semi ring of $\mathrm{P}_{\mathrm{T}}[\mathrm{x}]$.

Let $\mathrm{M}_{\mathrm{T}}[\mathrm{x}]$ be a polynomial sub ternary semiring of $\mathrm{P}_{\mathrm{T}}[x]$ which containing $\mathrm{S}_{\mathrm{T}}[x]$.

let $r \in \mathrm{K}_{\mathrm{T}}[x] \Rightarrow \mathrm{r}=r_{1} \cdot r_{2} \cdot r_{3} .---r_{n}$, where $r_{1}, r_{2}, r_{3},---, r_{n} \in \mathrm{S}_{\mathrm{T}}[x]$.

since $r_{1}, r_{2}, r_{3},---, r_{n} \in \mathrm{S}_{\mathrm{T}}[x]$ and $\mathrm{S}_{\mathrm{T}}[x]$ is contained in $\mathrm{M}_{\mathrm{T}}[x]$,

then $r_{1}, r_{2}, r_{3},---, r_{n} \in \mathrm{M}_{\mathrm{T}}[x] \Rightarrow \mathrm{M}_{\mathrm{T}}[x]$ is a polynomial sub ternary semiring.

Therefore $\mathrm{K}_{\mathrm{T}}[x]$ is the smallest polynomial sub ternary semiring of $\mathrm{P}_{\mathrm{T}}[x]$ containing $\mathrm{S}_{\mathrm{T}}[x]$.

Hence $\mathrm{P}_{\mathrm{T}}[x]$ is a polynomial ternary semiring generated by a polynomial sub ternary semiring $\mathrm{S}_{\mathrm{T}}[x]$.

\section{CONCLUSION}

In this paper mainly we studied about Polynomial ternary semirings. 


\section{ACKNOWLEDGMENTS}

Our thanks to the experts who have contributed towards preparation and development of the paper.

\section{REFERENCES}

[1] Dutta, T.K. and Kar, S., A Note on Regular Ternary Semirings, Kyung-pook Math. J., IV6 (2006), 357-365.

[2] Lehmer. D. H., A Ternary Analogue of Abelian Groups, Amer. J. Math., 59(1932), 329338.

[3] Madhusudhana Rao. D., Primary Ideals in Quasi-Commutative Ternary Semigroups International Research Journal of Pure Algebra - 3(7), 2013, 254-258.

[4] Madhusudhana Rao. D., Anjaneyulu. A., Gangadhara Rao. A., Prime $\Gamma$-Radicals in I-semigroup- International eJournal of Mathematics and Engineering 138 (2011) 12501259.

[5] Subramanyeswara Rao. VB., Anjeneyulu. A., Madhusudhana Rao. D., Partially Ordered $\Gamma$ semigroups-International Journal of Engineering Research \& Technology (IJERT) ISSN: 2278-0181, Vol. 1 Issue 6, August-2012.

[6] Sarala. Y., Anjaneyulu. A., Madhusudhana Rao. D., Ternary Semigroups-International Journal of Mathematical Science, Technology and Humanities 76 (2013) 848-859.

[7] Madhusudhana Rao. D., Srinivasa Rao. G., A Study on Ternary semirings-International Journal of Mathematical Archive-5 (12) December-2014, pp. 24-30.

[8] Vasantha. M., Madhusudhana Rao. D., Venkateswara Rao. M., Structure and Study of Elements in Ternary $\Gamma$-Semigroups-International Journal of Engineering Research, Volume 4, Issue 4, $1^{\text {st }}$ April-2015, pp 197-202.

[9] Siva Prasad. P., Madhusudhana Rao. D., Srinivasa Rao. G., A Study on Structure of POternary semirings- Journal of Advances in Mathematics, Volume 10, No 8, pp 3717-3724. 